\title{
Using a Physical Reference Frame to Study Global Circulation Variability
}

\author{
J. M. CASTANHEIRA \\ Department of Physics, University of Aveiro, Aveiro, Portugal \\ H.-F. GRAF \\ Max-Planck Institute for Meteorology, Hamburg, Germany \\ C. C. DACAMARA \\ Department of Physics, University of Lisbon, Lisbon, Portugal \\ A. ROCHA \\ Department of Physics, University of Aveiro, Aveiro, Portugal
}

(Manuscript received 16 January 2001, in final form 1 October 2001)

\begin{abstract}
The 3D structures of the free oscillations of an adiabatic and hydrostatic atmosphere around a basic state at rest were used as a physical filtering for atmospheric data. This filtering procedure allows for the consideration of the three primitive variables $(u, v, \phi)$ over the whole atmosphere simultaneously. Accordingly, the computed statistics do not simply rely on the information provided by a single variable of circulation, such as the 500$\mathrm{hPa}$ geopotential field.

Using this method, two classical patterns were isolated in the barotropic component of the circulation, one resembling the Pacific-North America (PNA) pattern, the other similar to the North Atlantic Oscillation (NAO) pattern in summer. Associating the barotropic and the second baroclinic components, a coupling in variability was retrieved between the strength of the winter stratospheric polar vortex and the tropospheric circulation over the North Atlantic. Until now these modes had only been recovered by means of statistical analysis. This study shows their existence in physically filtered fields.

The obtained results make clear that the observed winter pattern of NAO is not a simple variability mode of the atmosphere, but results instead from mean flow wave interaction that modulates tropospheric planetary Rossby waves.

The association between the NAO circulation variability patterns and the anomalies of the $850-\mathrm{hPa}$ temperature field was also investigated.
\end{abstract}

\section{Introduction}

The search for recurrent atmospheric circulation patterns is usually performed by means of a statistical analysis on gridded values of field variables (e.g., Wallace and Gutzler 1981; Preisendorfer 1988; Kushnir and Wallace 1989; Bretherton et al. 1992). However, in order to achieve statistically stable solutions, the number of degrees of freedom must be kept small. This means that one must consider a limited region of the atmosphere, that is, limited horizontal areas as well as a small number of vertical levels. Besides, the uncovered patterns are based on a purely statistical approach and their physical significance is usually tested, a posteriori, by the fraction

Corresponding author address: Dr. J. M. Castanheira, Dept. of Physics, University of Aveiro, 3810-193 Aveiro, Portugal. E-mail: jcast@fis.ua.pt of explained variability, by the significance level of the computed statistics, or by retrieving similar patterns from different subsets of the data (e.g., North et al. 1982; Livezey and Chen 1983; Kushnir and Wallace 1989).

Another type of approach consists in a prefiltering of data by means of a Fourier analysis allowing to isolate the most relevant zonal wavenumbers or by means of a spherical harmonic analysis aiming to select both the most important zonal wavenumbers and meridional scales (e.g., Schubert 1986; Nakamura et al. 1987; Perlwitz et al. 2000). The physical reason for using spherical harmonics comes from the fact that they are eigensolutions of the nondivergent barotropic vorticity equation over the sphere, with the same dispersion relationship of the Rossby-Haurwitz waves. [Spherical harmonics appear also as asymptotic forms of wave solutions of the linearized shallow water equations (Longuet-Hig- 
gins 1968).] The search for circulation patterns by means of an analysis performed in the phase spaces of either Fourier or spherical harmonics coefficients does bring some a priori meaning to the uncovered patterns due to the fact that the obtained statistics are computed on the amplitudes of functions that are believed to represent spatial structures of physical entities. However, the nondivergent barotropic vorticity equation does not account for the vertical stratification of the atmosphere and is certainly not an adequate approach for the intertropical circulation.

The motivation for this article lies on the assumption that the more physically based the entities are, from which statistics are derived, the more physical meaning may be assigned to the uncovered patterns.

The linearization of the atmospheric primitive equations around a basic state at rest is an oversimplification since it disregards the nonlinearity of the real atmosphere and does not account for a climatological wind. In spite of these important limitations, a set of linearized primitive equations does grasp much more of the physics of the real atmosphere than does the nondivergent barotropic vorticity equation. On the other hand, the normal modes - free oscillations - of the linearized system are vector functions defined over the whole atmosphere and represent, simultaneously, the horizontal wind and mass fields. This allows for the possibility of a dynamically consistent filtering of the atmospheric circulation (Daley 1991).

This article presents a method for the use of 3D atmospheric normal modes in the study of global atmospheric circulation variability. The method will be applied to the National Centers for Environmental Prediction (NCEP) reanalysis data covering the period 1959-99. A short description of the 3D normal modes of the linearized primitive equations will be given in section 2. The filtering of the atmospheric data by the projection onto the normal mode basis, and the most important variability patterns of the winter tropospheric and lower stratospheric circulations will be described in section 3. Section 4 will focus on the representation in the $3 \mathrm{D}$ normal scheme of the connection between the strength of the stratospheric polar vortex and the tropospheric circulation. Sections $4 \mathrm{a}$ and $4 \mathrm{~b}$ specifically address the association between two North Atlantic Oscillation (NAO)-like circulation variability patterns, here obtained, and the anomalies of the $850-\mathrm{hPa}$ temperature field. Concluding remarks will be given in section 5 .

\section{Linearized primitive equations}

A hydrostatic and adiabatic atmosphere can freely oscillate around a reference state at rest. The vertical and horizontal structures of each mode of oscillation can be separated, the horizontal structure being identical to that of a free oscillation mode of an incompressible, homogeneous, hydrostatic and inviscid fluid over a ro- tating sphere. For such an atmosphere, the primitive equations, linearized with respect to a basic state at rest having a pressure-dependent temperature distribution $T_{0}(p)$, may be written in the following form:

$$
\begin{aligned}
\frac{\partial u}{\partial t}-2 \Omega v \sin \theta+\frac{1}{a \cos \theta} \frac{\partial \phi}{\partial \lambda} & =0, \\
\frac{\partial v}{\partial t}+2 \Omega u \sin \theta+\frac{1}{a} \frac{\partial \phi}{\partial \theta} & =0, \text { and } \\
\frac{\partial}{\partial p}\left[\frac{1}{S_{0}} \frac{\partial}{\partial p}\left(\frac{\partial \phi}{\partial t}\right)\right]-\boldsymbol{\nabla} \cdot \mathbf{V} & =0,
\end{aligned}
$$

where $(\lambda, \theta, p)$ are the longitude, latitude, and pressure coordinates; $\phi$, the perturbed geopotential field, is the deviation from the basic state geopotential profile $\Phi_{0}(p)$; and

$$
S_{0}=\frac{R}{p}\left(\frac{k T_{0}}{p}-\frac{d T_{0}}{d p}\right)
$$

is the static stability parameter of the reference state. The remaining symbols in Eqs. (1) and (2) are the horizontal wind components $(u, v)$, the earth's radius $a$, the angular speed of earth's rotation $\Omega$, the specific gas constant $R$, and the ratio $k$ of specific gas constant to specific heat at constant pressure.

As model boundary conditions, it is assumed that $\omega$ $=d p / d t$ vanishes as $p \rightarrow 0$ and that the linearized geometric vertical velocity $w=d z / d t$ vanishes at a constant pressure $p_{s}$ near the earth's surface.

The free oscillations-normal modes-of the linearized primitive Eq. (1) may be written in the form,

$$
\left[\begin{array}{c}
u \\
v \\
\phi
\end{array}\right]_{\mathrm{msl}, \alpha}=\exp (-i 2 \Omega \nu t) G_{m}(p) \exp (i s \lambda) \mathbf{C}_{m} \cdot\left[\begin{array}{c}
U(\theta) \\
i V(\theta) \\
Z(\theta)
\end{array}\right]_{\mathrm{msl}, \alpha}
$$

where $\mathbf{C}_{m}=\operatorname{diag}\left[\left(g h_{m}\right)^{1 / 2},\left(g h_{m}\right)^{1 / 2}, g h_{m}\right]$ is a diagonal matrix of scaling factors, with $g$ the earth's gravity and $h_{m}$ the equivalent height. Here, $G_{m}(p)$ are the separable vertical structures and $m$ is a vertical index. The horizontal structures are given by the product of a zonal wave with wavenumber $s$ and a vector $[U(\theta), i V(\theta), Z(\theta)]_{\mathrm{msl}, \alpha}^{\mathrm{T}}$, which defines the meriodinal profiles of the wave. Because the meridional index $l$ is associated with the number of zeros of the meridional profiles, it may be regarded as an index of the meridional scale of the motion. The index $\alpha=1,2,3$ refers to westward traveling inertio-gravity waves, Rossby planetary waves, and eastward traveling inertio-gravity waves, respectively. Parameter $\nu$ is a dimensionless frequency.

The normal modes form a complete orthogonal basis that allow expansion of the horizontal wind and the geopotential fields (Cohn and Dee 1989; Tanaka 1994; Castanheira et al. 1999): 

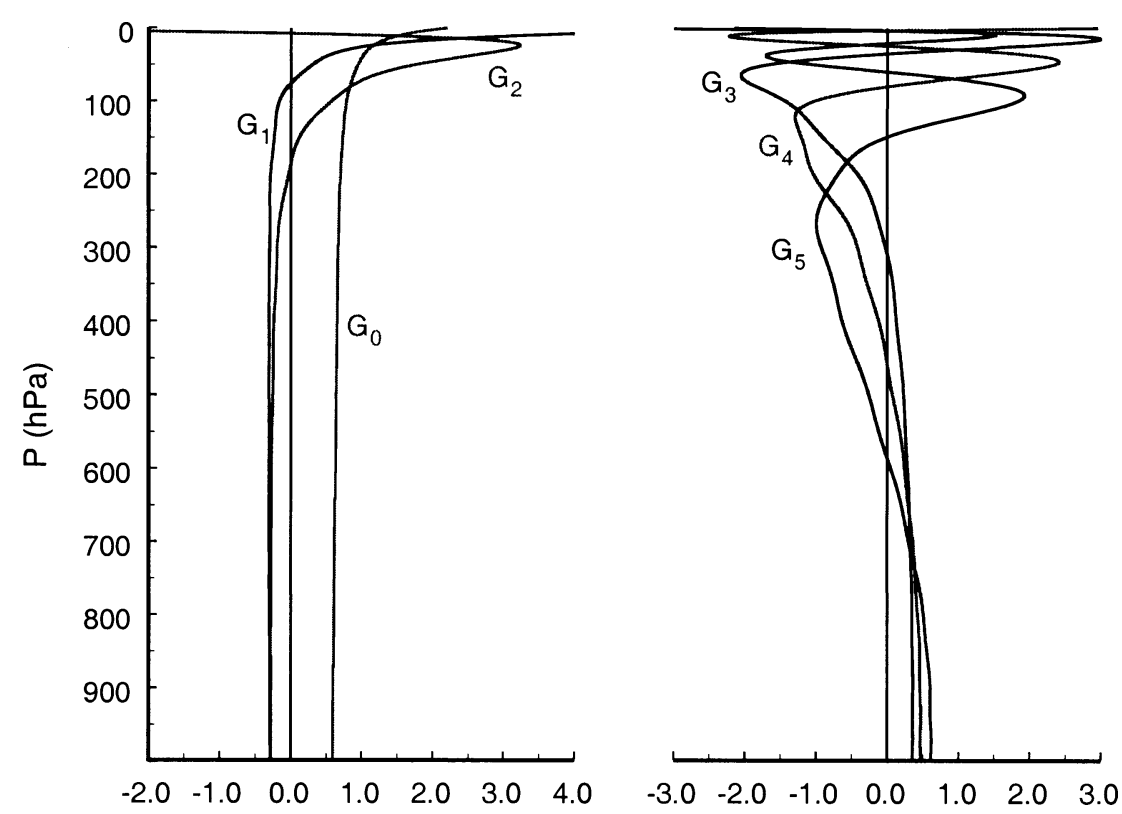

FIG. 1. Vertical structures of the modes $m=0, \ldots, 5$ of the NCEP atmosphere. The reference atmosphere was computed based on the DJF means from 1974 to 1996.

$$
\begin{aligned}
{\left[\begin{array}{l}
u \\
v \\
\phi
\end{array}\right]=} & \sum_{m=0}^{\infty} \sum_{s=-\infty}^{\infty} \sum_{l=0}^{\infty} \sum_{\alpha=1}^{3} w_{\mathrm{msl}}^{\alpha}(t) G_{m}(p) \exp (i s \lambda) \mathbf{C}_{m} \\
& \times\left[\begin{array}{c}
U(\theta) \\
i V(\theta) \\
Z(\theta)
\end{array}\right]_{\mathrm{msl}, \alpha} .
\end{aligned}
$$

The expansion coefficients are obtained by means of a vertical projection,

$$
(\hat{u}, \hat{v}, \hat{\phi})_{m}^{\mathrm{T}}=\frac{2}{p_{s}} \int_{0}^{p_{s}}(u, v, \phi)^{\mathrm{T}} G_{m}(p) d p,
$$

followed by a horizontal projection,

$w_{\mathrm{msl}}^{\alpha}=\frac{1}{2 \pi} \int_{0}^{2 \pi} \int_{-\pi / 2}^{\pi / 2}\left(\mathbf{H}_{\mathrm{msl}}^{\alpha}\right) * \mathbf{C}_{m}^{-1} \cdot(\hat{u}, \hat{v}, \hat{\phi})_{m}^{\mathrm{T}} \cos \theta d \theta d \lambda$.

We have assumed that the vertical structures, $G_{m}(p)$, and the horizontal structures, $\mathbf{H}_{\mathrm{msl}}^{\alpha}(\lambda, \quad \theta)=\exp (i s \lambda)$ $[U(\theta), i V(\theta), Z(\theta)]_{\mathrm{ms}, \alpha}^{\mathrm{T}}$, have unitary norms. The superscript $\mathrm{T}$ designates the transpose, and ( $)^{*}$ denotes the complex conjugate of the transpose.

It may be shown that the squared expansion coefficients are proportional to the total (i.e., kinetic + available potential) energy per unit area associated with the respective modes (Tanaka 1994; Castanheira et al. 1999)

$$
E_{\mathrm{msl}}^{\alpha}=\frac{p_{s} h_{m}}{c_{s}}\left|w_{\mathrm{msl}}^{\alpha}\right|^{2}
$$

For $s=0, c_{0}=8$ and $E_{m 0 l}^{\alpha}$ represents the total energy associated with a zonal symmetric $\alpha$ type mode with vertical and meridional indices $(m, l)$, respectively; whereas for $s \geq 1, E_{\mathrm{msl}}^{\alpha}$ represents the total energy of the complex conjugate pair of modes $(\alpha, \mathrm{msl})$ and $[\alpha$, $m(-s) l]$ and therefore $c_{s}=4$.

\section{Principal patterns of variability}

The data for this study were obtained from the NCEP reanalysis dataset (Kalnay et al. 1996). We used the winter monthly means [December-February (DJF)] of the vector $(u, v, \phi)^{\mathrm{T}}$, available at 17 standard pressure levels $(1000,925,850,700,600,500,400,300,250$, $200,150,100,70,50,30,20$, and $10 \mathrm{hPa}$ ) with a horizontal grid resolution of $2.5^{\circ}$ lat $\times 2.5^{\circ}$ lon, covering the period 1959-99. We have also considered the winter monthly means of the virtual temperature from 1974 to 1996, to compute the vertical profiles of both the basic state temperature $T_{0}(p)$ and the basic state stability parameter $S_{0}(p)$.

Figure 1 shows the first six vertical structures of the NCEP atmosphere. Because $G_{0}(p)$ has no node and is approximately constant throughout the troposphere, it is called the barotropic vertical structure. Each one of the other functions, $G_{m}(p)$ crosses the zero line $m$ times and is therefore referred to as the $m$ th baroclinic vertical structure. It is worth remarking that the ordering of the vertical baroclinic modes is based only on the number of crossings of the node line. The variance (transient total energy) associated with each vertical mode is not a monotonic decreasing function of the order of the mode.

In this paper we will focus our attention to the barotropic and the second baroclinic vertical structures. The projection of the atmospheric circulation $(u, v, \phi)^{\mathrm{T}}$ onto 


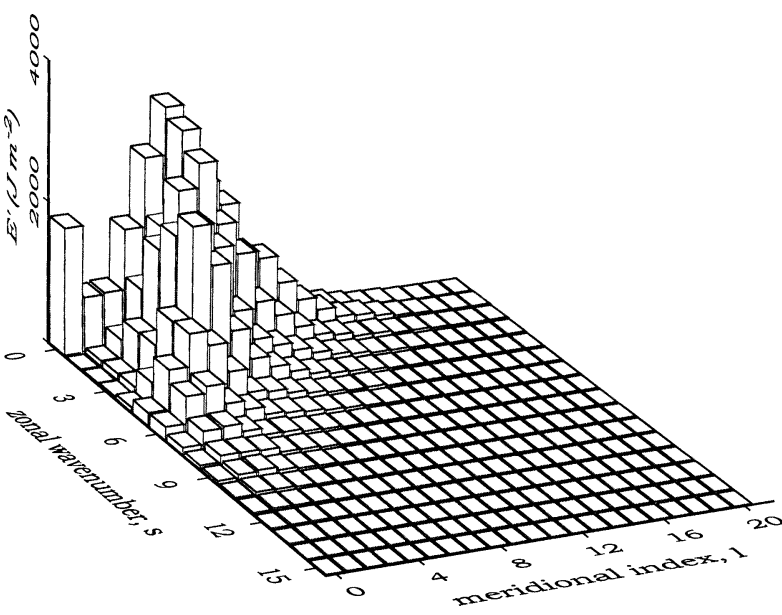

FIG. 2. Transient total energy associated with the barotropic Rossby modes and zonal Kelvin mode $(s=0, l=0)$ of the NCEP atmosphere.

the barotropic structure is strongly dominated by the contribution of the vertically averaged tropospheric circulation. We will consider this projection as representing the tropospheric circulation and refer to it either as barotropic or tropospheric.

The second baroclinic vertical structure, $G_{2}$, is negative with relatively small absolute values in the troposphere. In the stratosphere it has much higher positive values, with a strong maximum in the lower stratosphere around the 20-hPa level. Therefore, the projection onto this vertical structure will mainly respond to the features of the circulation located in the lower stratosphere. We will consider this projection as representing the lower stratospheric circulation and interchangeably refer to the classifications as second baroclinic or lower stratospheric. This vertical structure may also be viewed as expressing the "troposphere-stratosphere compensation principle," that is, a cold stratosphere overlying a warm troposphere and vice versa.

The first baroclinic vertical structure $G_{1}$, possess the highest weight above the $20-\mathrm{hPa}$ level. Since there is only one data level above $20 \mathrm{hPa}$, the vertical structure $G_{1}$ will not be discussed here.

\section{a. Tropospheric circulation}

Identification of those horizontal modes that are important to retain in the analysis of tropospheric circulation variability was performed by computing the variance of each barotropic coefficient $w_{0 s l}^{\alpha}$, since it is proportional to the transient total energy associated with the respective mode. By transient total energy we mean the total energy associated with the deviation of the monthly circulation field from the long-term mean. This is a very effective way of achieving a physically based filtering of the data. Figure 2 shows the horizontal energy spectrum of the barotropic Rossby modes. The gravity modes present a relatively very small variance (of the order of $10^{-3}$ of that of Rossby modes) and are, therefore, neglected. That means we only keep $\alpha=2$ in the analysis. The only exception is the zonal Kelvin mode, which is represented in Fig. 2 with zonal wavenumber $s=0$ and meridional index $l=0$. The variability of this mode is associated with an expansion or a contraction of the atmosphere, and respectively with larger or smaller values of the zonal mean zonal wind. The Kelvin mode is then to be expected as quite sensitive to anomalies of the heating field like those associated with El Niño. In fact, an ensemble experiment, performed with the Melbourne University atmospheric general circulation model (AGCM), revealed a strong sensitivity of the zonal Kelvin mode to the sea surface temperature (SST) anomalies (Castanheira 2000). Other modes with zonal wavenumbers $s>0$ did also show a very strong sensitivity to ENSO-related anomalies but in the case of the baroclinic vertical structures. It should be, however, emphasized that here we are only concerned with the barotropic vertical structure.

A visual inspection of Fig. 2 suggests to just retain in the analysis of the tropospheric circulation variability only those modes with wavenumbers $s<7$ and meridional indices $l<11$. In fact, these modes globally represent $89 \%$ of the total barotropic variance.

The horizontal patterns of variability were uncovered by means of a principal component analysis (PCA) performed on the time series of the $w_{0 s l}^{\alpha=2}$ coefficients selected in the previous step. These time series were previously detrended by subtraction of their 5-yr running means and it is worth noting that by using this simple detrending method the decadal variability has also been excluded. We ordered the detrended $w_{0 s l}^{\alpha=2}$ coefficients in a column vector

$$
\left[w_{1}^{\prime}(t), w_{2}^{\prime}(t), \ldots, w_{\beta}^{\prime}(t), \ldots, w_{q}^{\prime}(t)\right]^{\mathrm{T}},
$$

where $\beta$ stands for a quartet of indices $(0 s l, \alpha=2)$ and $q$ is the number of modes that were retained in the analysis. Next we computed the complex variance-covariance matrix

$$
\mathbf{S}_{\beta \beta^{\prime}}=\frac{1}{N-1} \sum_{t=1}^{N} w_{\beta}^{\prime}(t) w_{\beta^{\prime}}^{\prime *}(t),
$$

and then we solved the eigenvalue and eigenvector problem

$$
\sum_{\beta^{\prime}} S_{\beta \beta^{\prime}} e_{k}\left(\beta^{\prime}\right)=\lambda_{k} e_{k}(\beta) .
$$

All eigenvalues are real because the variance-covariance matrix $\mathbf{S}$ is Hermitian.

Finally, replacing the $w_{0 s l}^{\alpha=2}$ coefficients in the expansion (4) by the respective components $e_{k}(\beta)$ of the eigenvector $\hat{e}_{k}$ one retrieves the tropospheric circulation pattern associated with a global variance $\lambda_{k}$.

Figure 3 shows the patterns associated with the first two barotropic principal components (PCs). Since the circulation anomalies of these two patterns mainly occur in the extratropical northern atmosphere, we will focus 

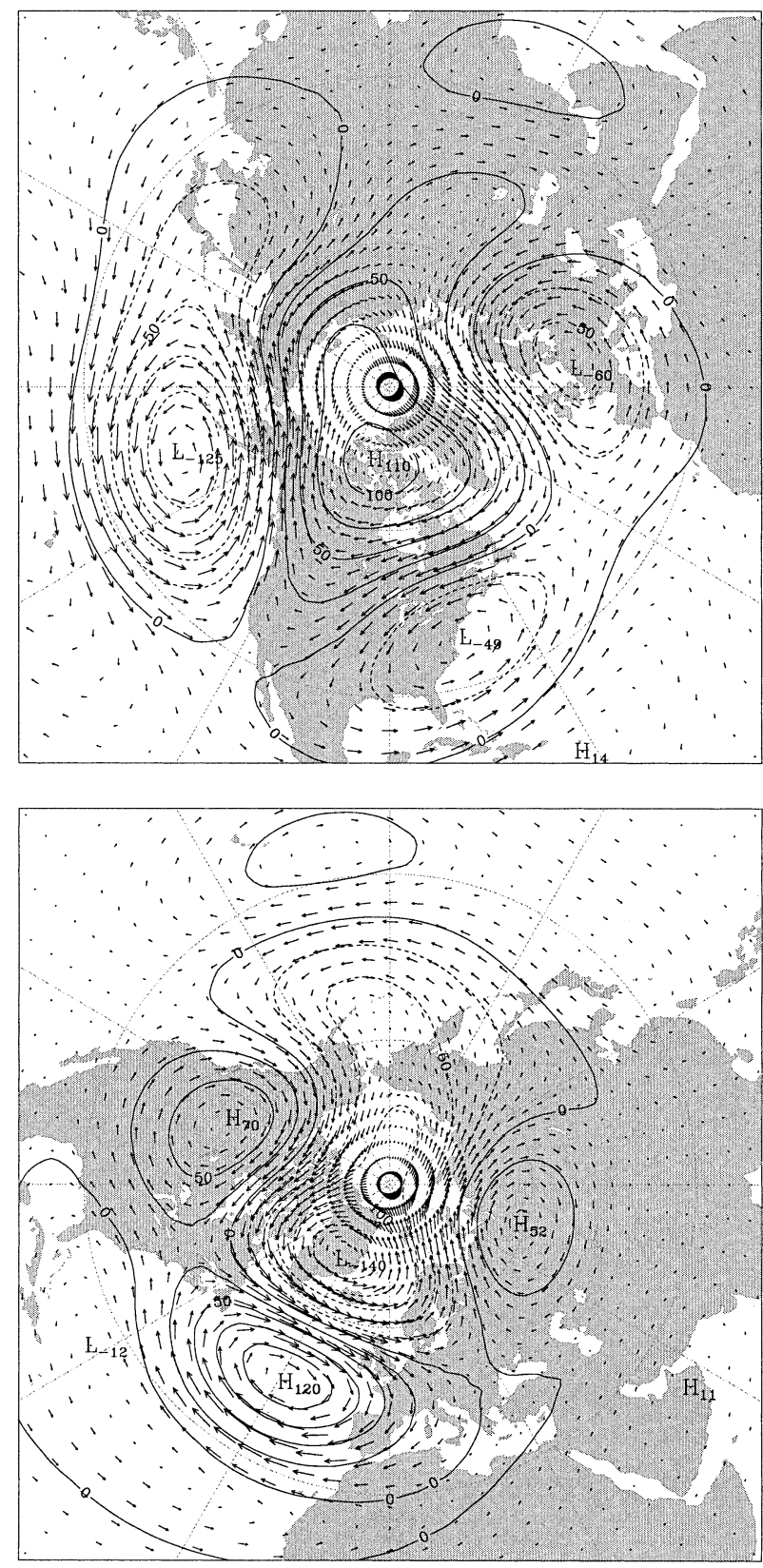

FIG. 3. (top) Patterns associated with the first PC ( $v_{\max }=9.6 \mathrm{~m}$ $\left.\mathrm{s}^{-1}, \sigma^{2}=13.4 \%\right)$ and (bottom) the second PC $\left(v_{\max }=11.6 \mathrm{~m} \mathrm{~s}^{-1}\right.$, $\left.\sigma^{2}=10.0 \%\right)$ of the tropospheric circulation. The geopotential and wind speed units are gpm and $\mathrm{m} \mathrm{s}^{-1}$, respectively.

on the Northern Hemisphere and show the most relevant features by means of stereographic projections. In fact this type of projection allows for a better resolution of the patterns as well as for easier comparisons with results from other authors. The first PC (PC1) explains $13.4 \%$ of the transient total energy of the global tropospheric circulation, and its associated pattern presents a wavelike structure over the North Pacific-North American sector that much resembles the Pacific-North America (PNA) teleconnection pattern (Wallace and

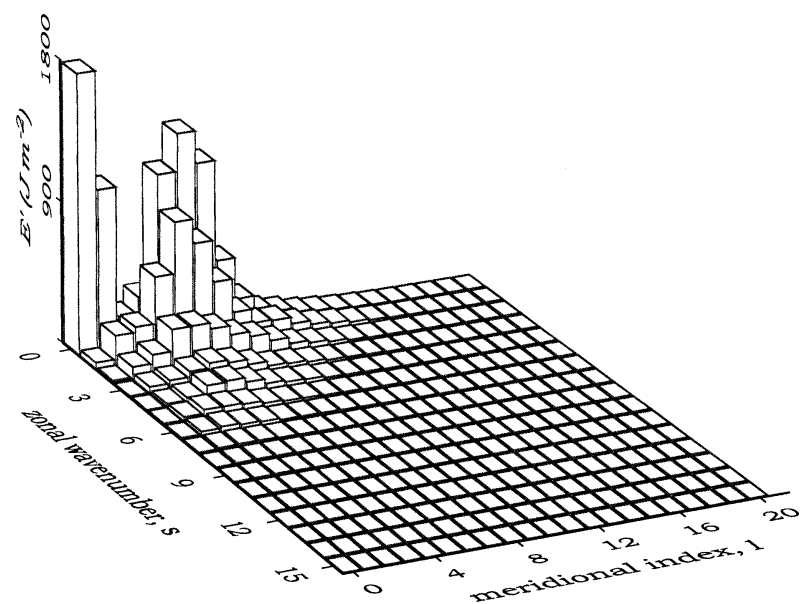

FIG. 4. Transient total energy associated with the Rossby and Kelvin $(s \leq 2, l=0)$ modes of the second baroclinic component of the NCEP atmosphere.

Gutzler 1981). In fact, the correlation between the PC1 and the PNA index time series [as defined by Wallace and Gutzler (1981)] is $r=0.78$.

The second PC (PC2) explains $10.0 \%$ of the variability and its associated pattern reminds the geopotential anomaly field associated with the cool-ocean-warmland (COWL) pattern (Wallace et al. 1996). However, its most prominent feature is the meridional dipole located over the central North Atlantic with stronger winds around the centers of the dipole. This dipolar structure is also shared by the NAO in summer (Glowienka-Hense 1990). The correlation between the detrended PC2 and a NAO index time series [defined as the difference between the normalized pressures at a station on the Azores (Ponta Delgada) and one on Iceland (Reykjavik)] is $r=0.60$. If we take the time series of the projections of the monthly nondetrended data, then the correlation with the NAO index increases to $r$ $=0.69$ (and for winter means, to $r=0.75$ ). However, we may notice that the typical winter "NAO pattern" is different. It has the centers of action shifted zonally (the high to the east and the low to the west). The relevance of this feature will be discussed in more detail in section 4 .

\section{b. Lower stratospheric circulation}

We proceeded with the lower stratospheric circulation in the same way as we did with the tropospheric one.

Figure 4 shows the horizontal energy spectrum for the second baroclinic vertical structure. The figure again represents the Rossby modes, excepting for the indices $(l=0 ; s=0,1,2)$ that stand for Kelvin modes. Once again the remaining gravity modes represent a relatively very small variance. As it may be observed in the figure there is an energy gap between the first and the fourth zonal Rossby modes that may be attributed to the fact that the Kelvin modes and the first Rossby modes rep- 

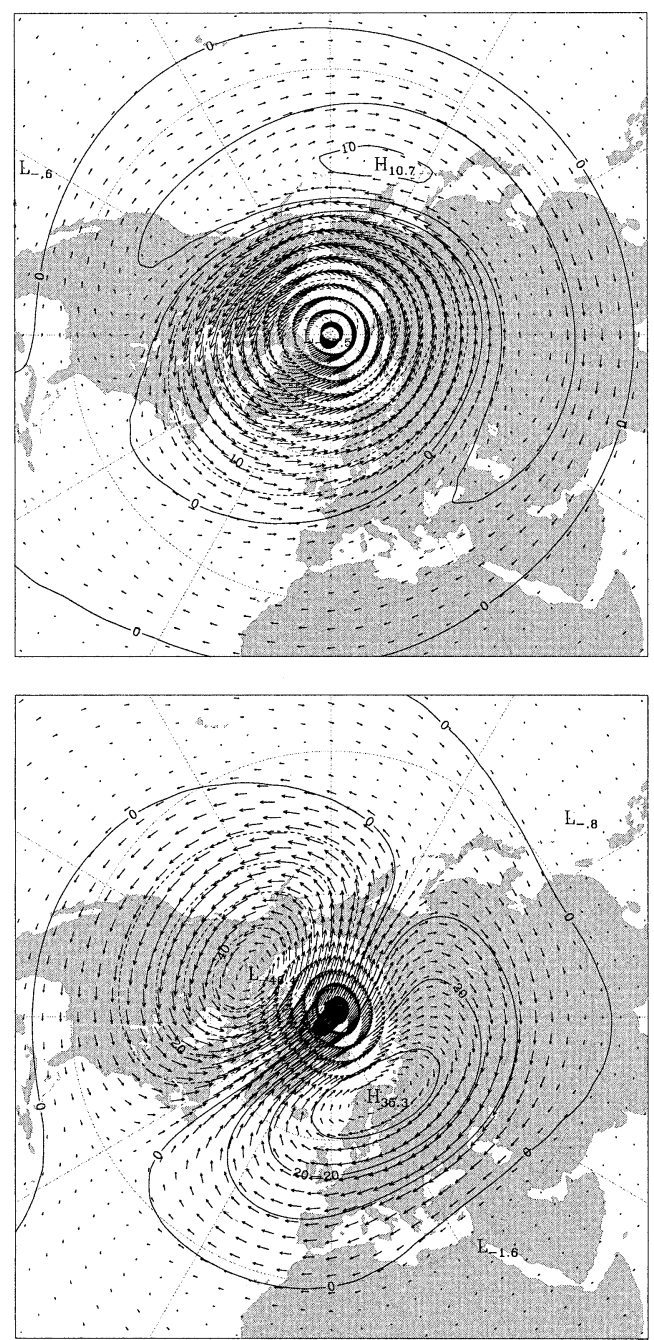

FIG. 5. (top) Patterns associated with the first PC ( $v_{\max }=1.9 \mathrm{~m}$ $\left.\mathrm{s}^{-1}, \sigma^{2}=34.8 \%\right)$ and (bottom) the second PC $\left(v_{\max }=2.1 \mathrm{~m} \mathrm{~s}^{-1}, \sigma^{2}\right.$ $=14.5 \%)$ of the lower stratospheric extratropical circulation.

resent intratropical variability whereas the Rossby modes with meridional index $l>3$ are linked to extratropical variability.

Results from Fig. 4 suggest retaining in the analysis of the stratospheric circulation variability only those modes with wavenumbers $s<5$ and meridional indices $l<11$. However, in order to avoid the problems related to the observations of the tropical circulation (Leder et al. 1998), we did retain the modes with meridional index $2 \leq l \leq 10$ for the extratropical circulation.

Figure 5 shows the patterns associated with the first two principal components of the lower stratospheric extratropical circulation. PC1 explains $34.8 \%$ of the retained variability, and represents the variability of the undisturbed stratospheric polar vortex. PC2 explains $14.5 \%$ of the variability and represents a wavenumber 1 disturbance of the polar vortex.

\section{Connection between stratospheric and tropospheric circulations}

From linear theory it is known that the vertical propagation of planetary waves into the stratosphere depends on the zonal mean zonal flow in the lower stratosphere, with a critical cut-off velocity that decreases with increasing wavenumber (Charney and Drazin 1961). This dynamical link between the tropospheric and stratospheric circulations manifests in a natural coupling between the variability of the two circulation fields. It is known that such natural coupling describes the connection between the strength of the stratospheric circumpolar vortex and a tropospheric circulation pattern, characterized by a hemispheric pressure seesaw between high and middle latitudes. In fact, the closest relationship may be found over the North Atlantic region with a NAO-like structure (Perlwitz and Graf 1995; Perlwitz et al. 2000). Other authors (Thompson and Wallace 1998, 2000) have obtained a more zonally symmetric tropospheric pattern when analyzing sea level pressure fields. This coupling of variability may also explain some of the recent trends of northern winter climate both observed (Perlwitz and Graf 1995; Thompson and Wallace 1998) and simulated (Shindell et al. 1999; Perlwitz et al. 2000). Therefore, such an important coupling must be well-defined and its correct simulation is crucial for answering to the still open question of the origin of the recent winter climatic trends.

The above-described natural coupling was found by canonical correlation analysis (Perlwitz and Graf 1995), singular value decomposition (Perlwitz et al. 2000) and regression analysis (Thompson and Wallace 1998) of the geopotential fields between pairs of one stratospheric level and one tropospheric level. In the works of Perlwitz and Graf (1995) and of Thompson and Wallace (1998) the statistical analysis was repeated for different tropospheric levels allowing to study the vertical structure of the mode. Therefore an improvement is to be expected in the physical understanding for this coupling of variability if it may be retrieved in the framework of 3D normal modes.

With the purpose of representing the coupling between the stratospheric polar vortex and the tropospheric circulation in the 3D normal mode scheme, we performed a multiple linear regression of the PC1 of the stratospheric circulation upon the PCs of the tropospheric circulation. The multiple linear regression is a special case of canonical correlation analysis, one in which we have only one variable in one of the two fields. Table 1 shows the single correlation coefficients between the PC1 of the stratospheric circulation and each one of the first $10 \mathrm{PCs}$ of the tropospheric circulation. Because the PCs of the barotropic component are orthogonal among themselves, the variance of the PC1 of the second baroclinic component, explained by the multiple linear regression, is given by the sum of the variances individually explained by each PC of the baro- 
TABLE 1. Correlation values between PC1 of the second baroclinic component and the first 10 PCs of the barotropic component. Values above the statistical significance level of $95 \%$ are marked with "*." The other values are below the $66 \%$ significance level.

\begin{tabular}{lccccccccccc}
\hline \hline & \multicolumn{10}{c}{ Barotropic component $(m=0)$} \\
\cline { 2 - 11 } & PC1 & PC2 & PC3 & PC4 & PC5 & PC6 & PC7 & PC8 & PC9 & PC10 \\
\hline PC1 $(m=2)$ & $0.50^{*}$ & $0.36^{*}$ & 0.05 & $0.34^{*}$ & -0.05 & $0.25^{*}$ & 0.04 & 0.09 & -0.01 & 0.03 \\
\hline
\end{tabular}

tropic component. Accordingly just PCs 1, 2, 4, and 6 of the barotropic component were retained in the multiple linear regression, resulting in a multiple correlation coefficient $R=0.75$.

Figure 6 shows the normalized canonical correlation pattern of the tropospheric circulation, given by

$$
\hat{\psi}=\frac{a_{1} \lambda_{1} \hat{e}_{1}+a_{2} \lambda_{2} \hat{e}_{2}+a_{4} \lambda_{4} \hat{e}_{4}+a_{6} \lambda_{6} \hat{e}_{6}}{\sqrt{\left(a_{1} \lambda_{1}\right)^{2}+\left(a_{2} \lambda_{2}\right)^{2}+\left(a_{4} \lambda_{4}\right)^{2}+\left(a_{6} \lambda_{6}\right)^{2}}}
$$

where $a_{k}$ are the regression coefficients and $\lambda_{k}$ are the variances associated with the eigenvectors $\hat{e}_{k}$. This coupled barotropic-baroclinic pattern explains $10.4 \%$ of the variability of the global tropospheric circulation.

A main feature of the obtained tropospheric circulation pattern is a northwestward tilted dipole between middle latitude northeast Atlantic and northwest Greenland, that is, a pattern that is known for winter NAO. This geopotential field drives a southwesterly wind from the North Atlantic into northern Europe. The advective transport by this wind must have a strong impact in the climate of northern Eurasia (Perlwitz and Graf 1995; Thompson and Wallace 1998). Hence, we find that the typical pattern of the winter NAO, as usually obtained from simple correlation analysis of gridded pressure data or by means of EOF analysis, is not the representation of an inherent atmospheric eigenmode. It is rather the result of the modulation of barotropic tropospheric eigenmodes by the strength of the stratospheric polar

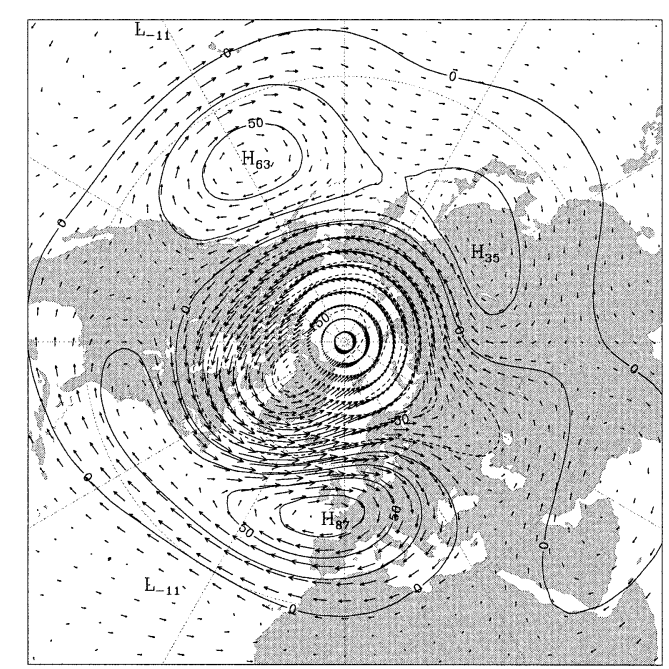

FIG. 6. Canonical correlation pattern of the tropospheric circulation associated with the strength of the stratospheric polar vortex $\left(v_{\max }=\right.$ $9.0 \mathrm{~m} \mathrm{~s}^{-1}, \sigma^{2}=10.4 \%$ ). vortex. This feature has important implications for climate variability and its simulation with climate models as will be discussed next.

\section{a. Lower troposphere temperatures and the normal modes}

Several studies (e.g., Graf et al. 1995; Shindell et al. 1999; Thompson et al. 2000) have shown that much of the observed temperature changes on the Northern Hemisphere of the last decades is in close connection with the value of the North Atlantic Oscillation, that is, with changes in the circulation over the North Atlantic. However, these results were mainly obtained on the basis of correlations of some kind of NAO indices, as derived from EOF-filtered data, with continental-scale temperatures. This type of analysis is not very sensitive to the actual patterns of variability because of the usage of very large-scale means. Here we will study how the two above-discussed variability patterns that present similarities with the NAO pattern (i.e., the second barotropic mode and the combination of the barotropic and the second baroclinic modes, Figs. 3, bottom; and 6, respectively) did contribute to the observed changes during the recent decades.

Accordingly we will first investigate the observed linearized trends of the temperature at the $850-\mathrm{hPa}$ level, that is, at the top of the planetary boundary layer for the last few decades (1959-99). This trend pattern was computed on the basis of the NCEP reanalysis data and is shown in Fig. 7. We have used the $850-\mathrm{hPa}$ temperature because it is more dependent on advective largescale processes than the near-surface atmosphere temperature. Near-surface temperature is affected by a manifold of processes at the surface (including those related to soil and vegetation types) that may not be completely captured either by the reanalysis scheme or by any of the current climate models.

The trend pattern in Fig. 7 is not at all regular and there is only little evidence for a COWL structure as it was suggested by Wallace et al. (1996) for near-surface air temperature. The isolines represent the linearized trends in $\mathrm{K} \mathrm{decade}^{-1}$, and the color shading indicates statistical significance (lowest limit at $95 \%$ confidence) of the linear trends. The main center of warming during the last decades is located over east Siberia, Mongolia, and northern China with $+4 \mathrm{~K}$ during the last $40 \mathrm{yr}$ at the maximum just east of Lake Baikal. Very strong warming has also occurred over central and southern Canada and parts of Alaska as well as in a band stretch- 


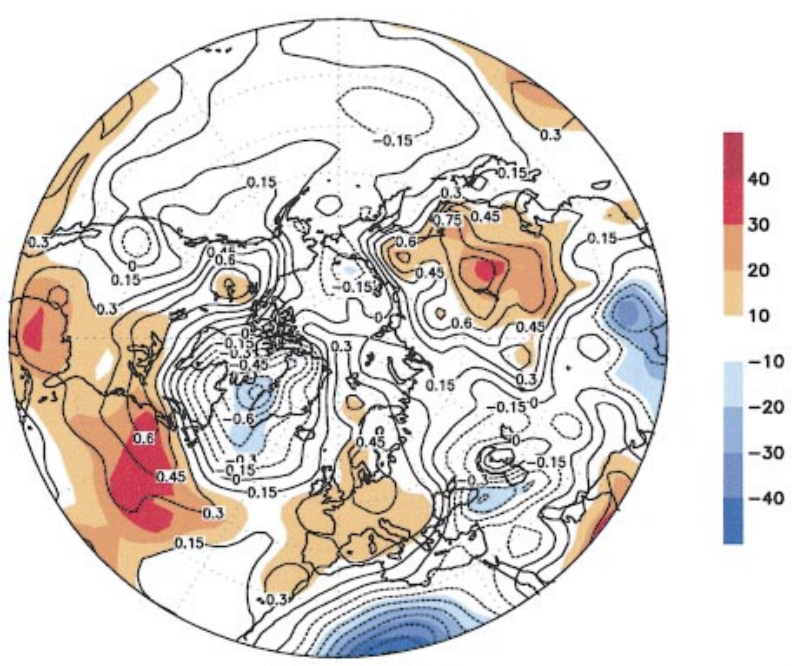

FIG. 7. Linear trend pattern of the winter $850-\mathrm{hPa}$ temperature field for the last 4 decades (1959-99). The isolines are the regression coefficients $\left(\mathrm{K} \mathrm{decade}^{-1}\right)$, and the colored areas are for percentage of explained variance (squared correlation coefficient). The red (blue) colors stand for positive (negative) trends.

ing along the $50^{\circ} \mathrm{N}$ circle of latitude across North America. A second significant Eurasian center of warming is found over western and central Europe with a maximum of close to $+2 \mathrm{~K}$ during the last four decades over Germany and southern Scandinavia.

Besides the warming areas, regions of large-scale cooling may also be found. In fact the lower troposphere became remarkably cooler in a region centered over the Davis Strait and including the eastern part of the Arctic Archipelago, Newfoundland, and Greenland. A higher level of statistical significance was only prevented by the high interannual variability over this region. Significant cooling is also apparent over the Sahara in North Africa as well as parts of the Middle East, parts of India, and Burma.

Next we discuss the temporal regression/correlation of the two NAO-type variability patterns with the 850$\mathrm{hPa}$ temperature field. It is worth noting that all time series that were considered in the computation of correlation-regression maps represent detrended data. The nondetrended time series of the expansion coefficients corresponding to the second barotropic and the combined barotropic-baroclinic patterns are given in Fig. 8. This figure also shows the respective $5-\mathrm{yr}$ running means, which were subtracted in order to obtain the detrended time series. A clear upward trend may be observed in the two time series.

Accordingly, we have used the temporal expansion coefficient of the second barotropic EOF based on detrended reanalysis data (Fig. 3) and performed a correlation-regression analysis with the detrended $850-\mathrm{hPa}$ temperature field (Fig. 9). Figure 9 is rather complex. The isolines indicate the regression coefficients, giving information on the amplitude of the temperature anom-
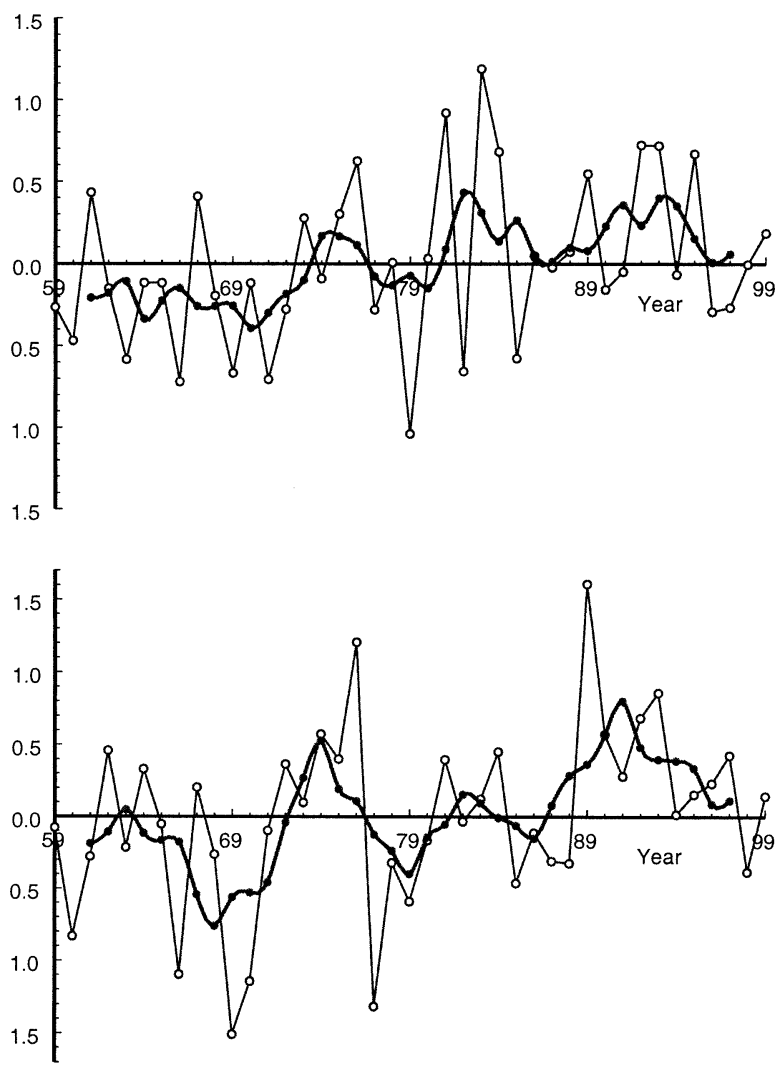

FIG. 8. (top) Winter mean projections of the nondetrended data onto the second barotropic detrended EOF and (bottom) onto the detrended barotropic-baroclinic regression pattern. The thick curves represent 5-yr running means.

aly fields. The color patterns show the explained fraction of total variance $\left(r^{2}\right)$ exclusively for those grid points where the correlation coefficient is statistically significant at least at the 95\% level. The red (blue) colors stand for positive (negative) correlations of T850 with the expansion coefficient. Figure 10 shows the analogous correlation-regression map for the temporal expansion coefficient of the combined barotropic-baroclinic variability pattern (Fig. 6). Although both figures do present some general similarities (like the strong negative correlation centered over the Davis Strait), clear differences are obvious as well. These features will be first discussed based on pattern correlations for different regions.

Table 2 shows the (area-weighted) spatial correlations between the T850 trend pattern for the last $41 \mathrm{yr}$ and the regression patterns for the temporal expansion coefficients of the second barotropic EOF and of the combined barotropic-baroclinic pattern (Figs. 9 and 10, respectively). The correlations are given for the whole area north of $40^{\circ} \mathrm{N}$ as well as for three subareas, that is, North Atlantic and Eurasia $\left(60^{\circ} \mathrm{W}-150^{\circ} \mathrm{E}\right)$, North Pacific and North America $\left(150^{\circ} \mathrm{E}-60^{\circ} \mathrm{W}\right)$, and Eurasia only $\left(0^{\circ}-150^{\circ} \mathrm{E}\right)$. In the last column of Table 2 , values of correlations are also given for more continental-scale- 


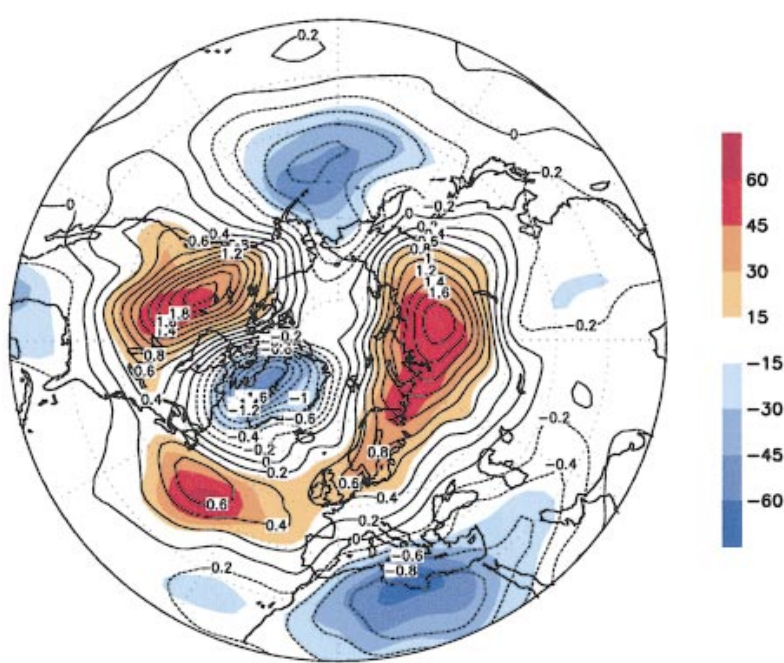

FIG. 9. Regression pattern of the detrended $850-\mathrm{hPa}$ temperature field onto the PC2 of the detrended barotropic circulation. The isolines are the regression coefficients (normalized to a std dev of the PC), and the colored areas are for percentage of explained variance. The red (blue) colors stand for positive (negative) correlations of T850 with the expansion coefficient.

based temperatures, that is, for the two longitudinal sectors $0^{\circ}-140^{\circ} \mathrm{E}$ and $60^{\circ}-125^{\circ} \mathrm{W}$ defined over the latitudinal band $40^{\circ}-70^{\circ} \mathrm{N}$. First column of Table 2 shows that if the Northern Hemisphere midlatitudes are considered without any further restrictions, then the expansion coefficients of both variability patterns give exactly the same correlation coefficients with the $850-\mathrm{hPa}$ temperature. On the other hand, for continental-scalebased temperatures (last column in Table 2) the pattern correlations with the second barotropic mode EOF are smaller than with the coupled barotropic-baroclinic pattern. If only the Eurasian part of the $\mathrm{NH}$ is considered (fourth column in Table 2) the second barotropic mode does only explain $5 \%$ of the trend pattern, whereas the coupled barotropic-baroclinic pattern captures about $41 \%$. It may therefore be concluded that barotropicbaroclinic pattern is able to explain a higher amount of the observed T850 trend pattern over Eurasia, whereas the capability of the barotropic mode to explain observed temperature variability appears to be concentrated more on the Western Hemisphere.

Figures 9 and 10 clearly show this behavior. Both correlation-regression patterns show a similar strong cooling tendency centered over the Davis Strait and,

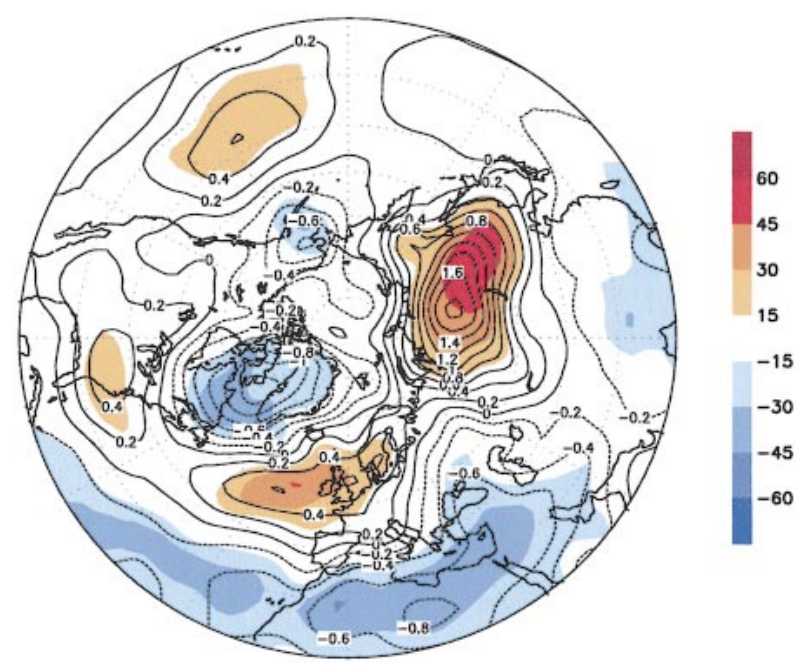

FIG. 10. As in Fig. 9, but w.r.t. the temporal expansion coefficient of the barotropic-baroclinic mode.

measured on a continental scale, warming tendencies over North America and Eurasia in mid- and high latitudes for positive PC values, but cooling in the subtropical area. However, the explained variance is much higher for the barotropic mode than for barotropic-baroclinic pattern over North America. Whereas, in accordance with the observed T850 trend pattern, the barotropic-baroclinic pattern has a double center over Eurasia (one over Europe, one over East Siberia and Mongolia), the barotropic mode regression pattern shows only one maximum concentrating over central Siberia. Differences may also be found in the correlation-regression patterns over the North Pacific. Whereas there is only a relatively small center of cooling over the western part of Alaska in the combined barotropic-baroclinic pattern, large-scale effects covering most of the central North Pacific north of $40^{\circ} \mathrm{N}$ may be found in connection with the barotropic mode. However, the observed trends (Fig. 7) do not show much of an effect in this area. Both the barotropic mode and the barotropic-baroclinic pattern do explain cooling in the subtropics but neither of them perfectly capture the observed trends. Whereas the barotropic mode explains more variability over North Africa, it does not describe the cooling over Southeast Asia as well as the barotropic-baroclinic pattern. In turn, the latter pattern em-

TABLE 2. Spatial correlation (explained variance, $r^{2}$ ) between the T850 trend pattern for the last $41 \mathrm{yr}$ and the T850 regression patterns for the temporal expansion coefficients of the second barotropic EOF and of the coupled barotropic-baroclinic pattern.

\begin{tabular}{|c|c|c|c|c|c|}
\hline Correlation area & North of $40^{\circ} \mathrm{N}$ & $\begin{array}{c}\text { North Atlantic } \\
\text { Eurasia } \\
\left(40^{\circ}-90^{\circ} \mathrm{N}\right. \\
\left.60^{\circ} \mathrm{W}-150^{\circ} \mathrm{E}\right)\end{array}$ & $\begin{array}{c}\text { North Pacific }+ \\
\text { North America } \\
\left(40^{\circ}-90^{\circ} \mathrm{N},\right. \\
\left.150^{\circ} \mathrm{E}-60^{\circ} \mathrm{W}\right)\end{array}$ & $\begin{array}{c}\text { Eurasia } \\
\left(40^{\circ}-90^{\circ} \mathrm{N},\right. \\
\left.0^{\circ}-150^{\circ} \mathrm{E}\right)\end{array}$ & $\begin{array}{c}\text { Continents only } \\
\left(40^{\circ}-70^{\circ} \mathrm{N},\right. \\
0^{\circ}-140^{\circ} \mathrm{E}, \\
\left.60^{\circ}-125^{\circ} \mathrm{W}\right)\end{array}$ \\
\hline Second barotropic PC & $0.64(41 \%)$ & $0.52(27 \%)$ & $0.81(66 \%)$ & $0.22(5 \%)$ & $0.53(28 \%)$ \\
\hline Coupled barotropic-baroclinic pattern & $0.64(41 \%)$ & $0.72(52 \%)$ & $0.46(21 \%)$ & $0.64(41 \%)$ & $0.66(44 \%)$ \\
\hline
\end{tabular}


phasizes a nonobserved cooling over the subtropical Atlantic.

\section{b. Discussion}

As noted above, the analysis of atmospheric variability on the basis of normal modes reveals that the pattern generally accepted as the NAO in Northern Hemisphere winter is not an eigensolution of the equations of motion. EOF analysis, that is normally used, depicts coherent patterns of variability and not physically based modes. However, the pattern of the second barotropic mode is very similar to the NAO pattern as obtained from simple correlation or EOF analysis in summer. This is especially important since it indicates that in summer-like conditions statistical stable solutions do capture a physical mode of variability. In summer the vertical propagation of planetary waves is blocked by the prevailing weak easterlies in the lower stratosphere. In winter the situation is different. On the one hand, vertical propagation of planetary waves may be also blocked if the westerlies are very strong in the lower stratosphere. Then, where vertical wave propagation is concerned, we are faced with a similar situation as in summer. On the other hand, if the westerlies are subcritical, then the waves can propagate to high altitudes. Therefore, in winter the strength of the polar vortex (in our analysis clearly described by the second baroclinic mode) modulates the behavior of the barotropic modes.

If we accept the paradigm (e.g., Corti et al. 1999) that forcing of the climate system will preferentially lead to the excitation of inherent variability modes (i.e., in our case of the eigensolutions of the equations of motion), the exact description of these modes is essential. The trend patterns of both the atmospheric circulation and of the lower tropospheric temperature during the winters of the last 4 decades can be much better described by the combined barotropic-baroclinic pattern than by the barotropic pattern alone. This implies a strong nonlinearity due to the existence of critical Rossby velocities for the vertical propagation of planetary waves.

The strength of the lower stratospheric westerlies has an impact on the interaction of tropospheric barotropic modes and determines the variability structure of tropospheric pressure fields and temperature. It is, therefore, not sufficient to study the influence of external forcing (like increase of greenhouse gases, volcanic eruptions, or ozone depletion) on the atmospheric circulation solely based on indices such as NAO. Rather, the interplaying modes need to be investigated. In our analysis we detected a total of four PCs of the barotropic and one of the second baroclinic modes that are relevant for the interpretation of observed circulation and lower tropospheric temperatures.

Graf et al. (1997) showed that the ECHAM4 model, mainly in its T21 resolution prefers the barotropic mode. Such preference manifests in the fact that more of the modeled variability is explained by the model's barotropic mode than of observed variability by the barotropic mode based on observations. The patterns of the leading coupled stratosphere-troposphere mode in this model are very similar to the second barotropic mode of this work (Fig. 3, bottom). This indicates that, other than in the real world, the climate model reacts to external forcing preferably with an excitation of barotropic structures and also that the modulation by the variability of the baroclinic mode (i.e., the strength of stratospheric westerlies) is only of secondary importance. The main reason of this behavior is the bias of climate models towards a too cold and too strong northern polar winter vortex.

Perlwitz et al. (2000) in their Fig. 5, showed patterns of the $850-\mathrm{hPa}$ temperature regressed on the temporal expansion coefficient of the 50-hPa singular pattern (the polar vortex strength) for weak and strong polar vortex conditions in a model simulation. Whereas the correlation-regression pattern we obtained for the observed data with the combined barotropic-baroclinic pattern (resembling best the observed trend pattern as discussed above) is dominant in a weak polar vortex regime in the model, the barotropic pattern is characteristic for the strong polar vortex regime. As most climate models, the ECHAM4-T42 model that Perlwitz et al. (2000) investigated has a bias toward a too strong and cold polar night vortex. Hence, the temperature changes observed during the last decades have a structure one may find when the climate model is in a regime where the cold polar bias is weak, or, alternatively, in a regime that is characteristic of the transition between a warm weak polar night vortex regime and a strong cold polar vortex regime. During such a transition period the effect of modulation of vertical planetary wave propagation is essential and the interference between barotropic and baroclinic modes is important. However, when the vortex becomes so strong that vertical propagation of planetary waves is prevented nearly all the time, the baroclinic mode (or the strength of the polar vortex) does not play a significant role anymore. Then, variability takes place preferably on the barotropic mode. Since Graf et al. (1998), Shindell et al. (1999), and Perlwitz et al. (2000) showed that the increasing greenhouse effect, possibly coupled to ozone depletion in early spring, will lead to an intensified polar vortex, we may expect for the future a change in the trend patterns towards the one for which the barotropic Rossby mode is responsible (Fig. 9).

\section{Summary and concluding remarks}

In this work a physical reference system was used to study the variability of the global atmospheric circulation. The mass and the horizontal motion fields were projected onto the free oscillations (normal modes) of a set of linearized primitive equations. Using this projection we were able to decompose the global circulation into one barotropic and several baroclinic components 
as well as into gravity-inertial and planetary (Rossby) waves. This procedure allows for a physically self-consistent filtering of the mass and the horizontal motion fields. Although the analyses were performed using global data, the discussion mainly focused on results for Northern Hemisphere winter.

The barotropic component was considered to represent the tropospheric circulation, the lower stratospheric circulation being represented by the second baroclinic component.

The variability patterns of the circulation were uncovered by means of a principal component analysis (PCA), performed in the phase space of the projections ( $w_{\mathrm{msl}}^{\alpha}$ coefficients). Only the Rossby and the Kelvin modes were shown to possess appreciable variability and were therefore retained in the analysis.

The first mode of variability of the tropospheric circulation (barotropic component) represents a PNA-like structure (Wallace and Gutzler 1981), and the second mode is similar to the summer NAO pattern (Glowienka-Hense 1990), but differs considerably from the observed winter NAO pattern.

The first PC of the lower stratospheric circulation (second baroclinic component) represents the variability of the undisturbed stratospheric polar vortex, and the second mode of variability represents a wavenumber 1 disturbance of the polar vortex.

We have also explored the concept of dynamical coupling between the tropospheric and the stratospheric circulations as known from linear wave theory (CharneyDrazin theorem). The connection between the stratospheric and tropospheric circulations was studied by means of a multiple linear regression between the strength of the polar vortex (PC1 of the lower stratospheric circulation) and the first 10 PCs of the tropospheric circulation. We refer to this coupling of variability between the two circulation fields as the barotropic-baroclinic pattern. The tropospheric circulation field of the latter pattern resembles the winter NAO pattern more closely than does the PC2-pattern of the barotropic (tropospheric) circulation. This result suggests that the observed winter NAO pattern results from the modulation of the barotropic Rossby modes by the stratospheric polar vortex. Most climate models present a too cold and strong polar winter vortex and therefore they will also have an important bias in the variability structure.

We have also studied the possibility of explaining the observed trend pattern in 850-hPa temperature (T850) either by the barotropic or by the coupled barotropicbaroclinic NAO-like patterns. The barotropic-baroclinic pattern explains a higher amount of the observed T850 trend pattern over Eurasia, whereas the barotropic mode explains a higher fraction of the trend over the Western Hemisphere. Comparing the obtained regression patterns of the T850 field upon the PC of each mode with the results of Perlwitz et al. (2000; see their Fig. 5), it may be stated that the regression pattern for the baro- tropic-baroclinic coupling (Fig. 10) resembles more closely the regression pattern they obtained for the weak polar vortex regime (their Fig. 5a). In turn, the regression pattern associated with the barotropic NAO mode (Fig. 9) resembles more closely the pattern for the strong polar vortex regime (their Fig. 5b). Since Graf et al. (1998), Shindell et al. (1999) and Perlwitz et al. (2000) showed that the increasing greenhouse effect, possibly coupled to ozone depletion in early spring, will lead to an intensified polar vortex, we may expect for the future a change in the trend patterns toward the one for which the barotropic Rossby mode is responsible (Fig. 9).

\section{REFERENCES}

Bretherton, C. S., C. Smith, and J. M. Wallace, 1992: An intercomparison of methods for finding coupled patterns in climate data. J. Climate, 5, 541-560.

Castanheira, J. M., 2000: Climatic variability of the atmospheric circulation at the global scale. Ph.D. thesis, University of Aveiro, Portugal, $186 \mathrm{pp}$.

, C. C. DaCamara, and A. Rocha, 1999: Numerical solutions of the vertical structure equation and associated energetics. Tellus, 51A, 337-348.

Charney, J. G., and P. G. Drazin, 1961: Propagation of planetaryscale disturbances from the lower into the upper atmosphere. $J$. Geophys. Res., 66, 83-109.

Cohn, S. E., and D. P. Dee, 1989: An analysis of the vertical structure equation for arbitrary thermal profiles. Quart. J. Roy. Meteor. Soc., 115, 143-171.

Corti, S., F. Molteni, and T. N. Palmer, 1999: Signature of recent climate change in frequencies of natural atmospheric regimes. Nature, 398, 799-802.

Daley, R., 1991: Atmospheric Data Analysis. Cambridge University Press, 457 pp.

Glowienka-Hense, R., 1990: The North Atlantic Oscillation in the Atlantic-European SLP. Tellus, 42A, 497-507.

Graf, H.-F., J. Perlwitz, I. Kirchner, and I. Schult, 1995: Recent northern winter climate trends, ozone changes and increased greenhouse forcing. Contrib. Atmos. Phys., 68, 233-248.

,-- , and - 1 1997: Coupled modes of tropospheric and stratospheric circulation in nature and in models. Stratospheric Processes and Their Role in Climate (SPARC), Proceedings of the First SPARC General Assembly, WCRP-99, World Meteorological Organization Tech. Rep. WMO/TD-814, 129-132.

- I. Kirchner, and J. Perlwitz, 1998: Changing lower stratospheric circulation: The role of ozone and greenhouse gases. J. Geophys. Res., 103 (D10), $11251-11261$.

Kalnay, E., and Coauthors, 1996: The NCEP/NCAR 40-Year Reanalysis Project. Bull. Amer. Meteor. Soc., 77, 437-471.

Kushnir, Y., and J. M. Wallace, 1989: Low-frequency variability in the Northern Hemisphere winter: Geographical distribution, structure and time-scale dependence. J. Atmos. Sci., 46, 31223142 .

Leder, S., K. Labitzke, and H. von Loon, 1998: Comparison between NCEP/NCAR and FUB analysis of the stratosphere. Proc. First WCRP Int. Conf. on Reanalysis, Silver Spring, MD, WCRP, World Meteorological Organization Tech. Rep. WMO/TD-876, 379-382.

Livezey, R. E., and W. Y. Chen, 1983: Statistical field significance and its determination by Monte Carlo techniques. Mon. Wea. Rev., 111, 46-59.

Longuet-Higgins, M. S., 1968: The eigenfunctions of Laplace's tidal equations over a sphere. Philos. Trans. Roy. Soc. London, A262, 511-607.

Nakamura, H., M. Tanaka, and J. M. Wallace, 1987: Horizontal struc- 
ture and energetics of Northern Hemisphere winter teleconnection patterns. J. Atmos. Sci., 44, 3377-3391.

North, G. R., T. L. Bell, and R. F. Cahalan, 1982: Sampling errors in the estimation of empirical orthogonal functions. Mon. Wea. Rev., 110, 699-706.

Perlwitz, J., and H.-F. Graf, 1995: The statistical connection between tropospheric and stratospheric circulation of the Northern Hemisphere in winter. J. Climate, 8, 2281-2295.

- - - and R. Voss, 2000: The leading variability mode of the coupled troposphere-stratosphere winter circulation in different climate regimes. J. Geophys. Res., 105, 6915-6926.

Preisendorfer, R. W., 1988: Principal Component Analysis in Meteorology and Oceanography. Elsevier, $425 \mathrm{pp}$.

Schubert, S. D., 1986: The structure, energetics and evolution of the dominant frequency-dependent three-dimensional atmospheric modes. J. Atmos. Sci., 43, 1210-1237.

Shindell, D. T., R. L. Miller, G. A. Schmidt, and L. Pandolfo, 1999:
Simulation of recent northern winter climate trends by greenhouse-gas forcing. Nature, 399, 452-455.

Tanaka, H. L., 1994: Normal mode energetics of the general circulation during the FGGE winter. University of Tsukuba, Institute of Geoscience Sci. Rep. 15A, 1-19.

Thompson, D. W. J., and J. M. Wallace, 1998: The Arctic Oscillation signature in the wintertime geopotential height and temperature fields. Geophys. Res. Lett., 25, 1297-1300.

- and - 2000: Annular modes in the extratropical circulation. Part I: Month-to-month variability. J. Climate, 13, 1000-1016. and G. Hegerl, 2000: Annular modes in the extratropical circulation. Part II: Trends. J. Climate, 13, 1018-1036.

Wallace, J. M., and D. S. Gutzler, 1981: Teleconnections in the geopotential height field during the Northern Hemisphere winter. Mon. Wea. Rev., 109, 784-812.

, Y. Zhang, and L. Bajuk, 1996: Interpretation of interdecadal trends in Northern Hemisphere surface air temperature. J. Climate, 9, 249-259. 